\title{
Design and Implementation of Novel Agricultural Remote Sensing Im- age Classification Framework through Deep Neural Network and Multi- Feature Analysis
}

\author{
YOUZHI ZHANG \\ Remote Sensing Technique Center of Heilongjiang Academy of Agriculture Sciences, \\ Heilongjiang 150086, China
}

Keyword: Agricultural Remote Sensing; Image Classification; Deep Neural Network; Feature Selection.

\begin{abstract}
With the rapid and bursting development of computer science and sensor technology, efficient remote sensing (RS) image classification algorithm is ur-gently needed. There are plenty of applications of re-mote sensing image processing techniques. In this paper, we propose a new agricultural remote sensing image classification and recognition method based on sparse auto-encoder deep neural network. Using an unsuper-vised learning algorithm features a large number of small pieces of sparse auto-encoder learning from some deep unlabeled images have already completed the training neural networks, and then learn features. The experiment and simulation prove the correctness of our model compared with other methods.
\end{abstract}

\section{INTRODUCTION}

\subsection{Background Survey}

With the rapid and bursting development of computer science and sensor technology, efficient remote sensing (RS) image classification algorithm is urgently needed. There are plenty of applications of remote sensing image processing techniques which could be categorized as the following parts. (1) RS image segmentation. In [1], Yuan et al. conducted research on feature analysis. They present segmentation solutions where representative features are either known or unknown. They also show that feature dimensions can be greatly reduced via subspace projection. (2) RS image super-resolution. In [2], Alfred conducted research on this topic. This presentation gives an overview of some essential steps. Class separability is accounted for by means of controlling the balance tuned by a smoothness parameter $\lambda$ between the prior and the likelihood terms in the posterior energy function. (3) RS image change detection. In [3], Badri's group conducted research on this issue, they pointed out that gibbs the proposed technique uses fuzzy markov random field model of spatial gray level properties of multispectral image difference. Change detection problem is solved using the maximum a posteriori probability estimation principle. (4) RS image registration. In [4] Li et al. introduces novel feature selection techniques to handle the problem. In addition, each key feature descriptor is refined to overcome different remote image of the gradient between the strength and direction. More applications such as de-haze and denoising techniques are also belonging to the usage of RS applications.

Remote sensing image classification is all like yuan according to the feature of the image is divided into several categories. Traditional image classification methods are mainly supervised and unsupervised classification, but the traditional unsupervised remote sensing image classification method is according to the data set of potential similarity clustering and appropriate measures, sometimes get better classification results, and supervised classification requires a lot of training data set to design the classifier, if the training data set is not enough to estimate classification parameters selected, often get better classification effect. More related research could be found in the literatures.

\subsection{Overview of Our Work}


Color remote sensing image classification based on SVM effect depends on the characteristics, is used to color the characteristics of remote sensing image classification has a lot of, the image color, texture feature is the most commonly used two kinds of feature vector. High resolution color remote sensing image contains abundant information, color is more similar to some of the area, but its texture feature is large, such as grass and forest land and some regional texture is similar, but the color is different, such as roads and bare land. And the color and texture are only part of the remote sensing image characteristics, the author in the process of classification, if you only use a single color or texture features to represent the image information, it does not fully describe the content of the image contains. To deal with this hardship, we propose the novel agricultural remote sensing image classification framework through deep neural network and multi-feature analysis.

\section{OUR PROPOSED METHODOLOGY}

\subsection{Overview of Deep Learning}

Since the code is a kind of unsupervised neural network learning algorithm, it makes the output value of the sample is equal to the input values. If the Numbers of hidden layer neurons is far less than neural network input layer and output layer, thus forcing the coding of neural network to learn the input data compression, said assumes that the neural network input data is completely random data, then, to learn these random data compression is very difficult; But if implied has a certain relationship between the input data of the specific structure, such as certain input characteristics are related to each other, so, the encoding algorithm can find the correlation between the related data, and to reconstruct the input data in the output layer. On the contrary, if the neural network hidden layer neurons number is more, or with the input and output layer is when, also can add a sparse sex of hidden layer neurons limit, so the encoding neural network can still find out the correlation between the input data.

Assuming that the activation of neurons function as the sigmoid function, when the output of the neuron is close to 1 that the neurons is active; Conversely, when the output of the neuron is close to zero that the neurons in the inhibitory state. Sparse sex of neural network, therefore, limit refers to most of the time limit neurons in the inhibitory state.

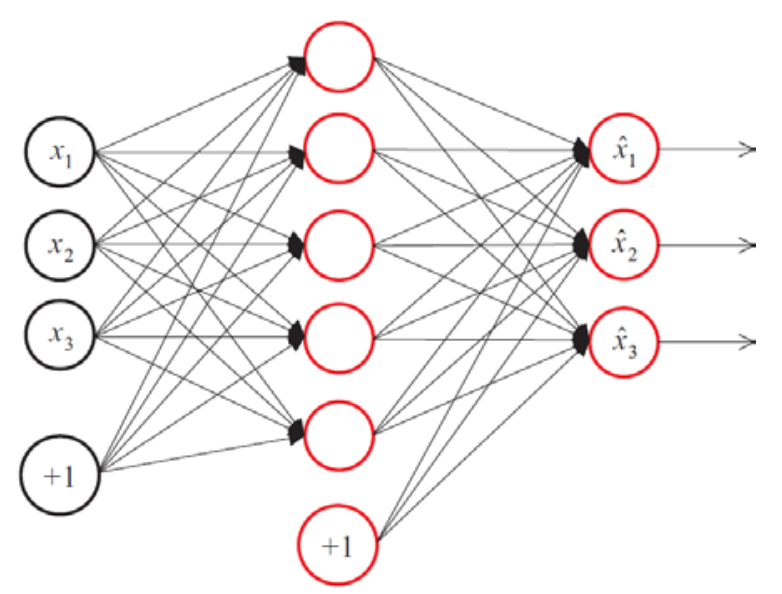

Figure 1.The structure of traditional neural network (TNN)

\subsection{The Deep Structure Analysis}

Bleaching is to reduce the phase correlation between the input image pixels, as many algorithms for preprocessing step. ZCA bleaching in this article, there are two main applications: the unmarked sample data preprocessing and learn the characteristics of the visualization. For a color remote sensing images, the arbitrary a pixel color information, and can be through the use of R, G, B value for combination said, therefore, by extracting each pixel of R, G, B value, can get the color information of image features. Color features should be defined clear, easy to extract, simple calculation, rela- 
tive to other features, color features is very stable. The conduction function could be expressed as the formula 1 .

$$
z_{i}^{(l+1)}=\sum_{j=1}^{S_{l}} w_{i j}^{(l)} x_{j}+b_{i}^{l}
$$

In the encoding neural network, still use BP algorithm and make no label sample input and output the results equal to target. Therefore, the unlabeled sample can be expressed as:

$\left\{x^{1}, x^{2}, \ldots, x^{i}, \ldots, x^{m}\right\}, 1 \leq i \leq m$

Through using $a_{j}^{(2)}\left(x^{i}\right)$ to denote the first set of sample input cases first $\mathrm{j}$ a hidden layer neurons of the output value. The average $\mathrm{j}$ neuron activation value is:

$\hat{\rho}_{j}=\frac{1}{m} \sum_{i=1}^{m} a_{j}^{(2)}\left(x^{i}\right)$

Bleaching is to reduce the phase correlation between the input image pixels, as many algorithms for preprocessing step. To join regularization weights in the regression model cost function attenuation after item, a new cost function is convex function, the existence of minimum feature learning algorithm since sparse coding is minimum cost function used when some of the iterative algorithm, such as batch gradient descent and Newton's method, LBFGS get the global optimal solution. Finally, wait for after the completion of the iterative algorithm and got the trained classifier. If the classifier output label is consistent with the test sample, show that classification results correctly, on the other hand, the classification result error; The statistical label test set classification correct sample amount divided by the total number of samples, get on the accuracy of image classification label test set. Therefore, the objective function could be revised to be the formula 4 .

$$
\begin{aligned}
& J(w, b)=\frac{1}{m} \sum_{i=1}^{m} \frac{1}{2}\left\|y^{i}-h_{w, b}\left(x^{i}\right)\right\|^{2}+ \\
& \frac{\lambda}{2} \sum_{l=1}^{n_{l}} \sum_{q=1}^{s_{l}} \sum_{p=1}^{s_{l+1}}\left(w_{p q}^{(l)}\right)^{2}+\beta \sum_{j=1}^{s_{2}} K L\left(\hat{\rho}_{j} \| \rho\right)
\end{aligned}
$$

In the formula 3, it is composed of three parts and, among them, the first part is the mean square error, the second part is the regularization item, last part is the penalty term relative entropy is a common method of measuring difference between two distributions. The depth of the neural network weight vector and the offset of the gradient descent direction vector can be expressed as:

$$
\left\{\begin{array}{c}
\nabla w^{(l)}=\frac{1}{m} \cdot \sigma^{(l+1)} \cdot\left(\sigma^{(l)}\right)^{T}+\lambda w^{(l)} \\
\nabla b^{(l)}=\frac{1}{m} \sum_{i=1}^{m} \sigma^{(l+1)}
\end{array}\right.
$$

As is shown in figure 2, 400 hidden neurons after training after the corresponding feature in the visual results, is divided into 20 columns show 20 lines, each character says of the hidden units learned in different position and direction of the image edge detection. 


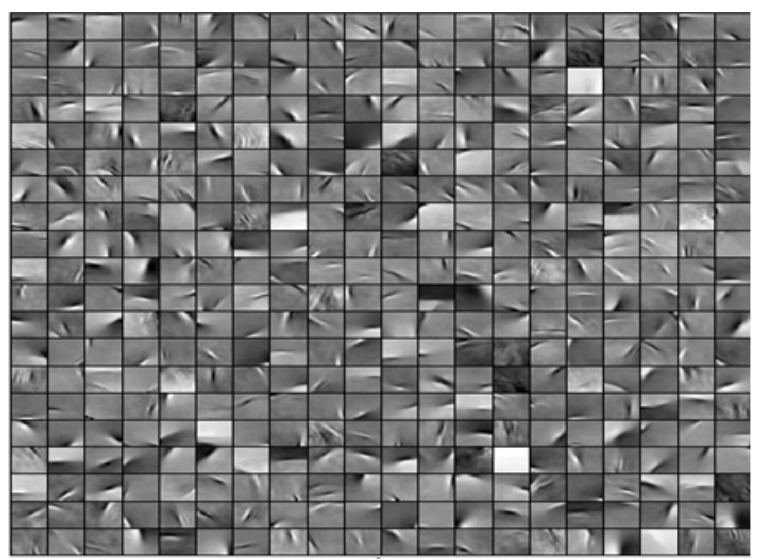

Figure 2.The learned feature through deep neural network (NDD) and overall selection procedure

\subsection{Multi-feature Analysis}

In remote sensing image classification, image texture feature is also a kind of commonly used feature vector. Commonly used texture characteristics are mainly texture features, gabor ripple and gray level co-occurrence matrix form. Of gray level co-occurrence matrix through the study of spatial gray level characteristics of image texture information, it can not only reflect the distribution of the brightness of the image features, but also can reflect the same or close to the brightness of the pixel location between distribution characteristics, and gradually become an important method used for analyzing the characteristic of image texture. The definition of gray level co-occurrence matrix is expressed as:

$$
P_{i j}=\frac{P_{\delta \theta, i j}}{\sum_{i=1}^{N 1} \sum_{j=1}^{N 2} P_{\delta \theta, i j}}
$$

The SVM algorithm based on texture feature must be used for image or has obvious phenomenon of texture regions. For those who have no obvious phenomenon of texture area or image, and the extraction of texture feature not only correctly describe the underlying characteristics of the image, and can lead to incorrect results. The color and texture features are combined together, therefore we can get a 7 division feature vector as follows:

$$
f=\left[R, G, B, W_{1}, W_{2}, W_{3}, W_{4}\right]
$$

Comprehensive features using the SVM classification algorithm combining the color features and texture features of images, it is not only applicable to all kinds of don't change color between images of the obvious, but also for all kinds of color information between don't close to the image, due to its combination of texture information, is still able to image for better classification. For a natural images, image within the scope of local statistical characteristics similar to those of other parts, so there's no need to let all the connections between input layer and hidden layer neurons, in order to reduce the neural network model parameters caused by the high resolution image of too much problem, convolution is applied in operation, so as to realize local to connect to the Internet, the basic idea is based on local connected network visual cortex of the brain neurons only response to the stimulation of some local area, only allow local connection neural network hidden layer neurons connected to the part of the input layer neurons. And for image texture feature is not obvious, because the combination of the color of the image information, still can for better classification, compared with the former two kinds of algorithm which more widely applicable. The corresponding steps could be visually described as the following figure 3. 


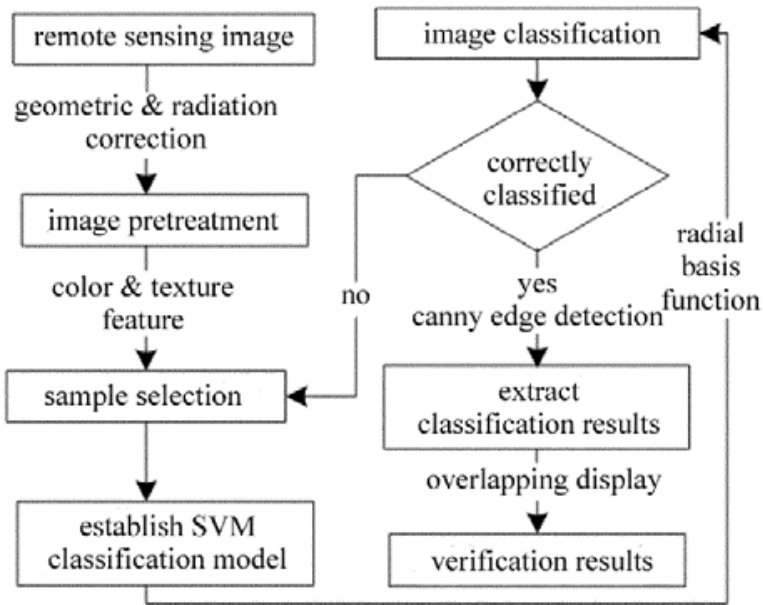

Figure 3.The description of our proposed method 


\section{EXPERIMENT AND SIMULATION}

\subsection{Set-up of the Experiment}

The simulation environment is initialized as the follows. Six physical machines equipped with 4 TB hard disk and 6 GB of RAM, and the simulation software is installed on Windows Win8 platform and Intel core 2 quad core $3.0 \mathrm{GHz}$ and $6 \mathrm{~GB}$ of RAM. Comprehensive features using the SVM classification algorithm combining the color features and texture features of images, it is not only applicable to all kinds of don't change color between images of the obvious, but also for all kinds of color information between don't close to the image, due to its combination of texture information, is still able to image for better classification. And for image texture feature is not obvious, because the combination of the color of the image information, still can for better classification, compared with the former two kinds of algorithm which more widely applicable.

\subsection{Simulation Result}

The following figures shows the result of classification using our method and other relate algorithms.

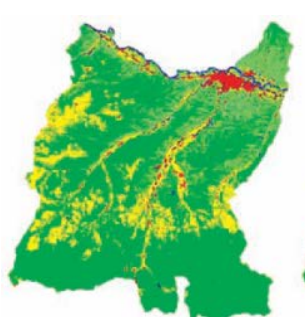

(1)

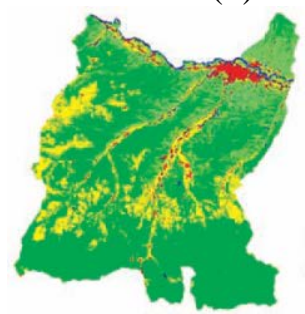

(4)

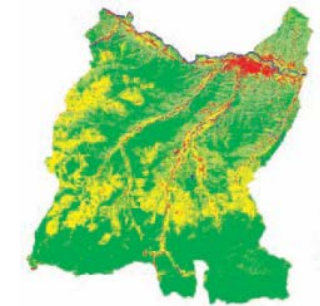

(7)

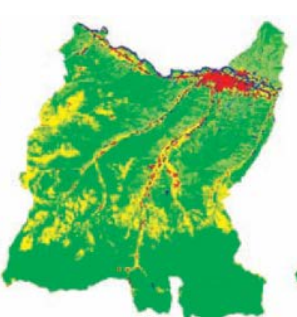

(2)

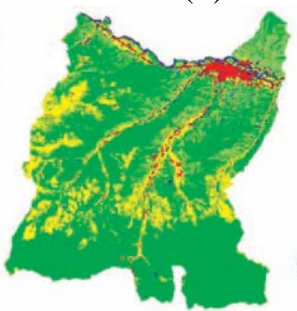

(5)

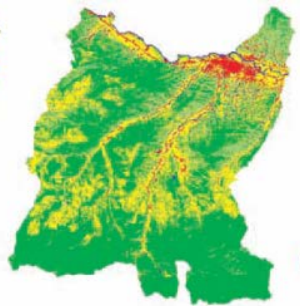

(8)

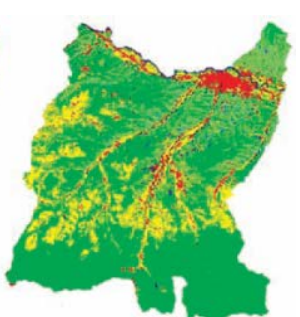

(3)

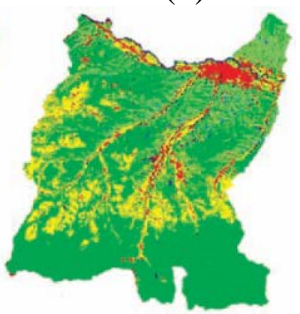

(6)

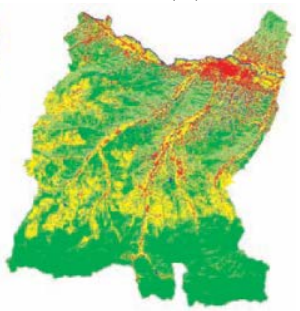

(9)

Figure 4.The experimental result of the research 


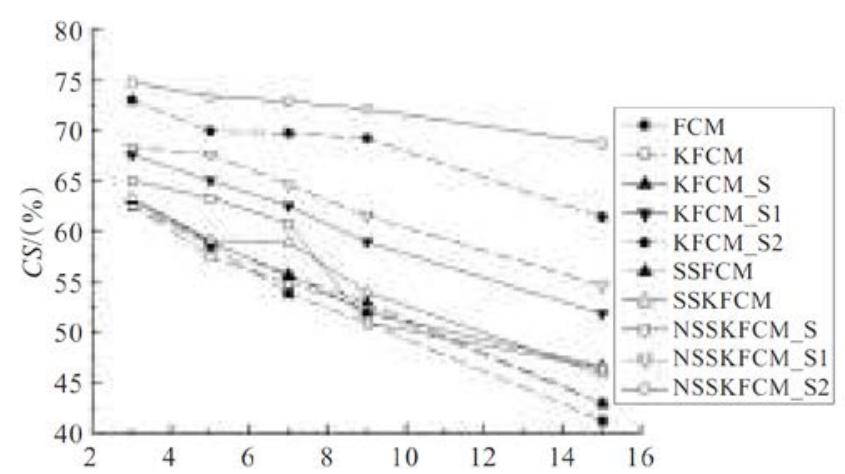

Figure 5.The statistical description of the simulation

\section{CONCLUSION AND SUMMARY}

With remote sensing image classification and recognition of agricultural problems and it is hard to distinguish between similar objects, this paper proposes a new agricultural remote sensing image classification and recognition method based on sparse auto-encoder deep neural network. Using an unsupervised learning algorithm features a large number of small pieces of sparse auto-encoder learning from some deep unlabeled images have already completed the training neural networks, and then learn features, features can be extracted from the massive images and winding and gathering. The experimental results show that the new method of image classification can be more effectively distinguish between forest fire and its object, similar to that of the red flag, red leaves, than the traditional neural network, etc.

In the future, we plan to refer to more related work to deal with the issue. The references we will combine are primary in the literatures [5-8].

\section{REFERENCE}

[1] Yuan, Jiangye, DeLiang Wang, and Rongxing Li. "Remote sensing image segmentation by combining spectral and texture features." Geoscience and Remote Sensing, IEEE Transactions on 52, no. 1 (2014): 16-24.

[2] Stein, Alfred. "Advanced remote sensing image analysis with super resolution mapping." In South African Symposium on Numerical and Applied Mathematics. 2015.

[3] Subudhi, Badri Narayan, Francesca Bovolo, Ashish Ghosh, and Lorenzo Bruzzone. "Spatiocontextual fuzzy clustering with markov random field model for change detection in remotely sensed images." Optics \& Laser Technology 57 (2014).

[4] Fatyga, Mirek, et al. "A comparison of three Deformable Image Registration Algorithms in 4DCT using conventional contour based methods and voxel-by-voxel comparison methods." Name: Frontiers in Oncology 5 (2015): 17.

[5] Mendoza, Niusvel Acosta, et al. "A Nectar of Frequent Approximate Subgraph Mining for Image Classification Un nectar sobre la minería de subgrafos frecuentes aproximados en clasificación de imágenes." Revista Cubana de Ciencias Informáticas 9.1 (2015).

[6] H. Wang and J. Wang, "An effective image representation method using kernel classification," in Tools with Artificial Intelligence (ICTAI), 2014 IEEE 26th International Conference on, Nov 2014, pp. 853-858.

[7] Ge, ZongYuan, et al. "Modelling Local Deep Convolutional Neural Network Features to Improve Fine-Grained Image Classification." arXiv preprint arXiv:1502.07802 (2015).

[8] Tran, Duc Toan, et al. "An approach for combining multiple descriptors for image classification." Seventh International Conference on Machine Vision (ICMV 2014). International Society for Optics and Photonics, 2015. 